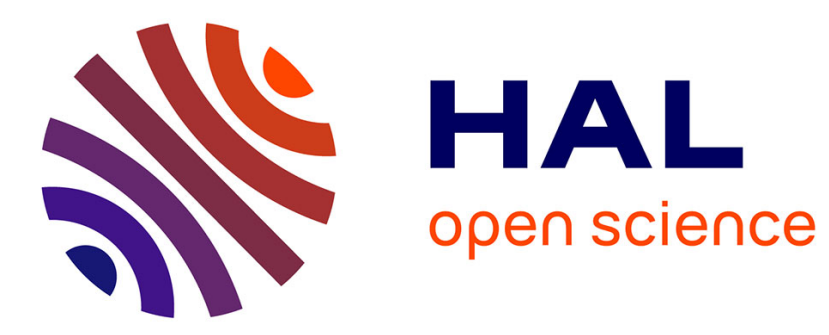

\title{
L'existence leibnizienne
}

Jean-Baptiste Jeangène Vilmer

\section{- To cite this version:}

Jean-Baptiste Jeangène Vilmer. L'existence leibnizienne. Archives de Philosophie, 2007, 70 (1), pp.249-273. halshs-00192973

\section{HAL Id: halshs-00192973 \\ https://shs.hal.science/halshs-00192973}

Submitted on 30 Nov 2007

HAL is a multi-disciplinary open access archive for the deposit and dissemination of scientific research documents, whether they are published or not. The documents may come from teaching and research institutions in France or abroad, or from public or private research centers.
L'archive ouverte pluridisciplinaire HAL, est destinée au dépôt et à la diffusion de documents scientifiques de niveau recherche, publiés ou non, émanant des établissements d'enseignement et de recherche français ou étrangers, des laboratoires publics ou privés. 


\title{
L'existence leibnizienne
}

\author{
Jean-Baptiste JEANGENE VILMER \\ EHESS / Université de Montréal / McGill University
}

$\rightarrow$ publié dans Archives de philosophie, 70:1, 2007, p. 249-273.

cet article est mis à la disposition du lecteur mais il ne correspond pas à la mise en page de la version définitive et publiée à laquelle il convient de se référer pour toute citation

RÉSUMÉ - Deuxième volet d'une étude consacrée à la possibilité et l'existence chez Leibniz, cet article tente de reconstruire la conception leibnizienne de l'existence, notion fondamentale qui soutient toute l'ontologie de l'auteur et qui n'est pourtant ni clairement définie ni systématisée. En trois parties, nous établissons que l'existence leibnizienne est bien un degré de possibilité, un ajout, un complément, mais elle n'ajoute rien de nouveau. Elle n'est pas une perfection, mais une relation comparative de perfections entre elles. Elle n'est pas un prédicat réel, mais un prédicat logique. Elle se définit comme ce qui est distinctement senti, ce qui plaît à un esprit tout en ne déplaisant pas à l'esprit le plus puissant, et ce qui est le plus harmonique. La conception leibnizienne de l'existence a plusieurs couches, et à de nombreux égards préfigure déjà la position kantienne.

ABSTRACT - Second part of a study dedicated to possibility and existence in the philosophy of Leibniz, this article attempts to reconstruct his conception of existence, a fundamental notion that supports the entirety of his ontology, which is nevertheless neither clearly defined, nor systematized. In three parts, we establish that Leibniz's conception of existence is a degree of possibility, an addition, a complement, which does not, however, add anything new. Existence is not a perfection, but a comparative relation of perfections between themselves. It is not a real predicate, but a logical predicate, characterized as that which is distinctively felt, as that which pleases a mind without displeasing the most powerful mind, and as that which is the most harmonious. Leibniz's conception of existence has several layers and already prefigures to many the position of Kant. 
L'ontologie leibnizienne est le chemin le plus court pour relier la possibilité à l'existence ${ }^{1}$. S'il fallait lui donner une forme géométrique, ce ne pourrait être le segment, car le point d'arrivée, l'existence, s'avère finalement aussi primitif que le point de départ, la possibilité. Leibniz, en effet, accorde la priorité à l'un comme à l'autre. Il soutient à la fois que, "de toutes les choses qui sont actuellement, la possibilité même ou impossibilité d'être est la première » et que «Je vis que celui qui aspire à trouver les principes des choses, devait commencer par la considération de l'existence : je me fatiguai des jours entiers à méditer sur cette notion de l'existence $»^{2}$. La possibilité et l'existence sont toutes deux des notions primitives, qui s'impliquent l'une l'autre. Si, chronologiquement, l'existence se manifeste avec plus de priorité, ontologiquement, c'est la possibilité qui est première, en tant qu'elle est la condition de possibilité de l'existence (l'existant est un possible existant). Ce chemin le plus court pour relier la possibilité à l'existence n'a donc pas l'allure d'un segment : c'est un cercle. Un cercle que l'on déplie pour les besoins de l'analyse.

En l'occurrence, c'est à ce bout qu'est l'existence qu'on s'intéresse ici, une notion fondamentale qui soutient toute l'ontologie leibnizienne et qui, pourtant, n'est ni clairement définie ni systématisée par l'auteur. Autant dire qu'elle appelle de la part de l'interprète un travail de reconstruction, d'autant plus utile qu'il faut, comme le conseille Leibniz, « commencer par la considération de l'existence ». Quelle est la conception leibnizienne de l'existence ? Peut-on seulement la définir ? A première vue, le philosophe de Hanovre semble soutenir qu'en tant que notion primitive, l'existence ne peut être définie : «Existens definiri non potest ${ }^{3}$. C'est, dit-il, une notion simple et inanalysable: «Existentia ergo est notio incomposita, sive irresolubilis $»^{4}$. Pourtant, à plusieurs reprises, les textes leibniziens présentent des définitions de l'existence ou, du moins, de l'existant. Cette notion primitive a priori indéfinissable est, dans les faits, définie. En revanche, elle ne l'est pas toujours clairement, d'où la nécessité de l'éclaircissement. S'il est faux de soutenir, comme le fait Jalabert, que «Leibniz ne définit pas l'existence », il faut reconnaître avec M. de Gaudemar que « la notion d'existence n'est pas clairement définie par Leibniz luimême » ${ }^{5}$.

Utilisant ce paradoxe, la plupart des commentateurs se montrent particulièrement sévères avec Leibniz sur ce point : pour Adams, par exemple, si l'existence est indéfinissable, c'est a posteriori parce qu'aucune des définitions données par l'auteur n'est consistante avec la théorie leibnizienne de la création ${ }^{6}-1$ 'indéfinissabilité de la notion apparaissant alors comme la conséquence de l'échec de Leibniz, qui curieusement serait aussi celui de ne pas présenter de définition unifiée de l'existence,

\footnotetext{
${ }^{1}$ Cet article est le deuxième moment d'une étude en trois parties sur la possibilité et l'existence chez Leibniz. Il succède à "Possibilité et existentiabilité chez Leibniz», Revue philosophique de Louvain, 104:1, 2006, p. 23-45. Il précède « De la possibilité à l'existence : Kant critique de Leibniz », Dialogue : Revue canadienne de philosophie, à paraître.

${ }^{2}$ Respectivement lettre à Foucher de 1676, GP I 370, 12, et texte supposé être de 1666, cité par Foucher de Careil, Mémoire sur la Philosophie de Leibniz, Paris : Rudeval, 1905, t. I, p. 10.

${ }^{3}$ Leibniz, Textes inédits d'après les manuscrits de la Bibliothèque provinciale de Hanovre, Gaston Grua (ed.), Paris : PUF, 1948, p. 325.

${ }^{4}$ De iis quae per se concipiuntur, septembre 1677, GP I 271.

${ }^{5}$ Respectivement J. JALABERT, «Les Notions d'Essence et d'Existence dans la Philosophie de Leibniz », Akten des Internationalen Leibniz-Kongresses, Hannover, 14-19 November 1966, Band I, Metaphysik - Monadenlehre, Studia Leibnitiana Supplementa, vol. I, Wiesbaden : Steiner, p. 14 et M. de GAUdemar, De la puissance au sujet, Paris : Vrin, 1994, p. 42, n. 1, souligné par nous (spn).

${ }^{6}$ Voir R. M. AdAMS, Leibniz : Determinist, Theist, Idealist, New York, Oxford : Oxford University Press, 1994, p. 164-176.
} 
puisqu'il n'en va pas de même pour Dieu et pour les créatures ${ }^{7}$. Tandis que pour Hacking, qui suit Curley, il n'y a tout simplement pas de «doctrine leibnizienne de l'existence » digne de ce nom ${ }^{8}$. Au contraire, nous trouvons que, non seulement l'auteur donne parfois des définitions explicites, claires et satisfaisantes, mais encore laisse-t-il ici et là à son lecteur de quoi reconstruire, de lui-même, une conception cohérente de l'existence - ne serait-ce qu'en négatif de la possibilité ou essence, car l'existence est au moins ce qui se détache de l'horizon des possibles ${ }^{9}$. Et nous l'allons montrer, en trois étapes.

\section{I- L'existence est-elle un degré de possibilité, un ajout, un complément de possibilité ?}

Parce que l'existence n'est pas un concept statique apparu ex nihilo, mais le résultat d'un processus continu depuis la possibilité, la question se pose naturellement de savoir si on peut alors la considérer comme un degré de possibilité, un ajout, un complément. C'est même concernant l'existence la question centrale.

Il faut pour répondre préciser les termes de la question. Il est évident, d'abord, que Leibniz considère l'existence comme un degré de possibilité (le plus haut, en l'occurrence), qu'il se demande ce qu'il faut ajouter à la simple possibilité pour atteindre l'existence (compossibilité et maximum), et qu'il parle même d'un « complementum possibilitatis », notamment dans la définition des forces primitives : «Aristote les appelle entéléchies premières, je les appelle peut-être plus intelligiblement forces primitives, qui ne contiennent pas seulement l'acte ou le complément de la possibilité, mais encore une activité originale $»^{10}$. Que l'existence soit, d'une manière générale, un degré de possibilité (« tout bien réfléchi, je ne vois pas qu'on puisse concevoir autre chose dans existant qu'un certain degré d'être ${ }^{11}$ ), un ajout (« nous concevons l'existence comme actuelle, c'est-à-dire comme quelque chose qui est ajouté à la possibilité ou à l'essence ») $)^{12}$, un complément, et quelque

\footnotetext{
${ }^{7}$ R. M. ADAMS, op. cit., p. 170, présente en effet la différence entre les deux définitions de l'existence comme un échec : « the obvious disadvantage of failing to present a unified account of the nature of existence ». L'existence de Dieu étant nécessaire, et celle de ses créatures contingente, nous ne voyons pas en quoi le fait d'avoir deux définitions distinctes de l'existence, pour coller à l'évidente différence de nature entre Dieu et ses créatures, peut constituer un quelconque défaut.

${ }^{8}$ Voir E. M. CURLEY, « The Root of Contingency », in H. G. Frankfurt (ed.), Leibniz : A Collection of Critical Essays, New York : Anchor Books, 1972, p. 69-97.

${ }_{9}$ « Existence, exister, cela veut dire étymologiquement sortir de, existere, sortir soit du royaume du possible, soit de l'absolu. Existe ce qui se détache sur l'ensemble des possibles ou ce qui se détache de l'absolu » (J. WAHL, Discussions sur l'idée d'existence, leçons à la Sorbonne, des 9 décembre 1939, 16 décembre 1939 et 6 janvier 1940, BNF, ref. : FOL-Z WAHL PIECE-1).

${ }^{10}$ Système nouveau de la nature et de la communication des substances, GP IV 479, in Leibniz, Système nouveau de la nature et de la communication des substances, et autres textes 1690-1703, C. Frémont (ed.), Paris: Flammarion, 1994, p. 67, spn. Voir par exemple J. WAHL, op. cit., p. 5 : «Leibniz se demande ce qui s'ajoute à la possibilité pour faire l'existence et il admet l'idée d'un complementum possibilitatis » et J.-B. RAUZY : «L'existence peut être conçue comme le plus haut degré de possibilité dans la mesure où la plus grande classe de compossibles serait choisie par un esprit. Cette définition de l'existence est sans doute un écho d'un texte parisien, où Leibniz écrivait : «Est harmonique au plus haut point ce qui est le plus plaisant pour l'esprit le plus parfait » (A VI iii 476, 11 février 1676) » (in Leibniz, Recherches générales sur l'analyse des notions et des vérités. 24 thèses métaphysiques et autres textes logiques et métaphysiques, J.-B. Rauzy (ed.), Paris : PUF, 1998 (ciaprès : Rauzy), p. 316, n. 74).

${ }_{11}^{11}$ Generales Inquisitiones de Analysi Notionum et Veritatum (1686), 73, in Rauzy, p. 247, spn.

${ }^{12}$ Generales Inquisitiones de Analysi Notionum et Veritatum (1686), 73, in Rauzy, p. 247-249, spn.
} 
chose de plus, en somme (" l'existant est toujours un être, c.-à-d. un possible, et quelque chose de plus $»^{13}$ ), voilà donc qui semble ne pas faire problème.

La véritable question ne se pose pas au niveau du genre, mais de l'espèce : l'existence ajoute à l'essence, mais quelle est la nature de sa contribution ? S'agit-il d'ajouter quelque nouveauté? Non, par définition, car si elle est un degré de possibilité, c'est qu'il n'y a pas entre elle et la possibilité le saut de nature qu'impliquerait une telle nouveauté. Et non, de toute façon, car l'existence n'est qu'une exigence d'essence, en elle l'existentiabilité est contenue : «Si l'existence était autre chose qu'une prétention de l'essence, alors elle aurait elle-même une essence et quelque chose de nouveau viendrait s'ajouter aux choses, à propos de quoi on pourrait à nouveau se demander si cette essence existe ou n'existe pas et pourquoi celle-ci plutôt que celle-là $»^{14}$. L'existence est donc à la fois l'ajout et ce à quoi l'on ajoute : en tant qu'ajout elle est en acte, et en tant que ce à quoi l'on ajoute, c'est-àdire en tant qu'essence, elle est en puissance ${ }^{15}$.

\section{II- L'existence est-elle une perfection ou, plus largement, un prédicat ?}

\section{A/ L'existence est-elle une perfection?}

Nous savons que l'existentiabilité de chaque possible est en proportion de sa perfection, c'est-à-dire de sa réalité. La question de savoir si, pour Leibniz, l'existence elle-même est une perfection, se pose donc naturellement à l'interprète. Pour autant, les lectures sur ce point sont divisées, comme semblent l'être les textes de l'auteur luimême. L'existence est-elle une perfection ? Cette question est délicate, et exige une acuité particulière. On peut distinguer deux approches, selon que la perfection est entendue comme prédicat de premier ordre, comme c'est le cas dans les preuves de la possibilité de l'existence de Dieu, ou comme prédicat du second ordre, c'est-à-dire en tant que propriété de propriété, lorsqu'elle est définie classiquement comme «degré ou quantité de réalité ou d'essence ».

A première vue, il paraît ne faire aucun doute que l'existence, pour Leibniz, est une perfection. Il semble effectivement la compter au nombre des perfections de Dieu, et l'on cite volontiers pour en témoigner le passage suivant : « (...) or l'idée de cet être enferme toutes les perfections, et l'existence en est une, par conséquent il existe $\|^{16}$. C'est oublier que l'auteur ici ne parle pas en son nom mais en celui de « quelques Philosophes anciens et nouveaux [qui] ont bâti une certaine démonstration de Dieu, qui est fort imparfaite ${ }^{17}$. Il ne fait, en somme, qu'exposer la preuve ontologique de l'existence de Dieu, celle de saint Augustin (philosophe ancien), revisitée par Descartes (philosophe nouveau), ce dernier étant la cible explicite des

\footnotetext{
${ }^{13}$ Generales Inquisitiones de Analysi Notionum et Veritatum (1686), 73, in Rauzy, p. 247.

${ }^{14}$ GP VII 195, note marginale.

${ }^{15}$ On trouve effectivement chez Leibniz une équivalence entre existence et actualité : «Petrus est abnegans actu, seu abnegans existens» ("Pierre est reniant en acte, c.-à-d. reniant existant»), Recherches générales sur l'analyse des notions et des vérités, 71, in Rauzy, p. 247. Si l'utilisation des termes aristotéliciens est utile et éclairante, il faut se garder d'extrapoler et de conclure que le passage de la possibilité à l'existence chez Leibniz n'est autre que celui de la puissance à l'acte chez Aristote : les concepts ne se recoupent pas proprement, comme le montre M. de GAUDEMAR, op. cit., I.

${ }^{16}$ Meditationes de Cognitione, Veritate et Ideis, II, 23, GP IV 449. C'est ce que fait A. HerreraIbáñez qui en déduit que « existir es para Leibniz une perfección » (A. HERRERA-IBAÑEZ, " Existencia, propiedad, cualidad, accidente y atributo en Leibniz», Revista de Filosofia (Universidad Iberoamericana, Mexico), 19:57, p. 422, n. 2).

${ }^{17}$ Meditationes de Cognitione, Veritate et Ideis, II, 23, GP IV 449.
} 
Meditationes de Cognitione, Veritate et Ideis. Cet extrait, comme beaucoup d'autres, ne peut donc prouver que Leibniz souscrit à la thèse cartésienne, utilisée à l'occasion de l'argument ontologique, selon laquelle l'existence est une perfection.

On s'attache alors à d'autres passages, où l'auteur, en son nom cette fois, déclare par exemple «Il est aussi certain que l'existence est une perfection, ou accroît la réalité, que, quand un existant $A$ est conçu, davantage de réalité est conçue que quand un possible $A$ est conçu ${ }^{18}$. L'erreur, ici, serait probablement de prendre Leibniz au mot. A contextualiser ce passage, qui appartient à la correspondance avec Eckhard, largement consacrée à cette question de savoir si l'existence est une perfection, on note que l'auteur, précédemment, était beaucoup moins catégorique. Peu avant, Leibniz doutait effectivement que l'existence puisse être une perfection, puisque les perfections sont des qualités, tandis que l'existence n'en est pas une : «Videri enim perfectiones esse quasdam qualitates, qualis non est existentia ${ }^{19}$. A cet endroit on comprend que la question de savoir si l'existence est une perfection dépend finalement de celle de savoir si elle est une qualité, c'est-à-dire un prédicat - ce que nous examinerons dans la section suivante.

Par ailleurs, il faut également noter l'imprécision terminologique et l'ambiguïté de l'expression «Il est aussi certain que l'existence est une perfection, ou accroît la réalité » : Leibniz ne concède à l'existence d'être une perfection qu'en tant qu'être une perfection, c'est accroître la réalité. Ce qu'il veut dire, en somme, c'est que l'existence accroît la réalité. Or la perfection, à proprement parler, n'est pas l'accroissement de la réalité (concept dynamique), mais la quantité ou le degré de réalité (concept statique) : l'auteur approuve la définition de la perfection comme « degré ou quantité de réalité ou d'essence ${ }^{20}$ que lui propose Eckhard, et soutient cette définition classique de la perfection de manière constante dans ses écrits. Il ne faut donc pas le prendre au mot lorsqu'il se laisse aller, parfois, à une formule ambiguë.

Ainsi, parce que l'existence n'est pas une quantité (statique) mais un accroissement (dynamique) de réalité, nous soutenons que l'existence n'est pas une perfection à strictement parler. Son caractère dynamique, qui la distingue de la perfection, s'explique par le fait que c'est en elle, de manière analytique, que l'existence contient l'existentiabilité, la tendance à l'existence. Ce résultat, cependant, doit rendre compte $\mathrm{du}$ fait que la perfection, indubitablement, reste le principe de l'existence : «Et ut possibilitas est principium Essentiae, ita perfectio seu Essentiae gradus (per quem plurima sunt compossibilia) principium existentiae $»^{21}$. Comment comprendre, dès lors, la nature de la relation qui lie l'existence à la perfection ?

L'existence n'est pas une perfection, mais une relation comparative de perfections entre elles, c'est-à-dire un accroissement de perfection, conformément à son caractère dynamique. C'est ce qu'établit de manière solide un texte peu connu et qui mérite d'être repris intégralement :

«Valde dubitari potest, an existentia sit perfectio, seu gradus realitatis : quoniam dubitari potest an existentia sit ex eorum numero quae concipi possunt seu ex partibus essentiae : an vero sit tantum quidam conceptus imaginarius qualis est caloris et frigoris, qui non est

\footnotetext{
${ }^{18}$ «Patet etiam Existentiam esse perfectionem, seu augere realitatem, id est, cum concipitur A existens, plus realitatis concipi, quam cum concipitur A possibile » (Lettre à Arnold Eckhard, de l'été 1677, GP I 266, spn).

${ }^{19}$ Colloquium cum Dno. Eccardo Professore Rintelensi Cartesiano, praesente Dni. Abbatis Molani fratre, 5 avril 1677, GP I 214.

${ }^{20}$ Correspondance avec Eckhard, GP I 225.

${ }^{21}$ De rerum originatione radicali, GP VII 304.
} 
nisi perceptionis nostrae denominatio, non rerum naturae. $\mathrm{Si}$ tamen accurate consideremus nos aliquid amplius concipere cum cogitamus rem $A$ existere, quam cum cogitamus esse possibile, ideo videtur verum esse, existentiam esse gradum quendam realitatis ; vel certe esse aliquam relationem ad gradus realitatis ; non est autem existentia aliquis realitatis gradus : nam de quolibet realitatis gradu intelligi potest tum possibilitas tum existentia : erit ergo existentia excessus graduum realitatis rei unius, supra gradus realitatis rei oppositae, id est id quod est perfectius omnibus inter se incompatibilibus existit et contra quod existit est caeteris perfectius. Itaque verum quidem est id quod existit perfectius esse non existente, sed verum non est, ipsam existentiam esse perfectionem, cum sit tantum quaedam perfectionum inter se comparatio. ${ }^{22}$

L'existence est-elle une perfection? Répondre par la négative est du même coup rapprocher Leibniz de ce qui sera la solution intermédiaire kantienne, qui refusera à l'existence d'être une perfection pour lui avoir refusé d'être un prédicat, en vertu précisément de ne lui avoir reconnu qu'un caractère relationnel : « le petit mot est n'est point un prédicat de plus, mais seulement ce qui pose le prédicat en relation avec le sujet $»^{23}$. Montrer que le caractère relationnel de l'existence se trouve déjà chez Leibniz, c'est montrer comment, contre les nombreux interprètes qui l'opposent à Kant sur ce point et sur tant d'autres ${ }^{24}$, le philosophe de Hanovre anticipe déjà la solution kantienne.

\section{B/ L'existence est-elle un prédicat ?}

Pendant longtemps, à la suite de Russell, les interprètes ont soutenu que, pour Leibniz, l'existence était un prédicat. Ainsi en est-il de B. Mates qui écrit : « existence appears to be a predicate for Leibniz - at least, he treats it as such in the only place where he explicitly considers the matter », ce qu'il justifie entre autre par le fait que l'existence est une perfection: "Also, at A.6.6.437, in connection with the ontological argument for the existence of God, he accepts Descartes's treatment of existence as a perfection. Cf. A.6.6.411 and G I 223, where existence is a quality of God ${ }^{25}$. Or, on vient de voir à quel point cette lecture était trop rapide, décontextualisée et imprécise : l'existence pour Leibniz n'est pas une perfection.

D'autres allèrent même plus loin, en utilisant cette conviction comme une raison suffisante de ranger Leibniz parmi les essentialistes, par opposition aux existentialistes; ainsi en est-il de Jalabert, qui prolonge Couturat: «La pensée moderne oppose aux philosophies de l'essence les philosophies de l'existence. On range parmi les premières la philosophie de Leibniz. (...) Certes, il y a chez Leibniz priorité de l'essence. L'existence d'une chose doit pouvoir se déduire a priori de l'essence. L'existence est un prédicat ${ }^{26}{ }^{2}$. Or il est possible de montrer comment Leibniz peut être beaucoup plus légitimement rangé parmi les existentialistes, si l'on tient toujours à cette distinction artificielle que pour notre part nous abandonnons volontiers $^{27}$. En somme, de telles lectures sont périmées.

\footnotetext{
${ }^{22}$ Existentia, an sit perfectio, 1677 (?), A VI-4-B 1354, spn.

${ }^{23}$ I. KANT, Critique de la raison pure, A 599 / B 627, in Euvres philosophiques, F. Alquié (ed.), Paris : Gallimard, 1980, t. I, p. 1215.

${ }^{24}$ Voir par exemple K. R. SEESKIN, «Is existence a perfection? - A case study in the philosophy of Leibniz », Idealistic Studies, 8:1, p. 124-135.

${ }^{25}$ B. MATES, The Philosophy of Leibniz. Metaphysics and Language, New York - Oxford : Oxford University Press, 1986, respectivement p. 101 et p. 101, n. 47.

${ }^{26}$ J. JALABERT, op. cit., p. 13, qui renvoie à Couturat, La logique de Leibniz, chap. VI.

${ }^{27}$ Voir notre article « Possibilité et existentiabilité chez Leibniz », Revue philosophique de Louvain, 104:1, 2006, p. 23-45.
} 
Aujourd'hui, l'interprétation contraire semble gagner du terrain, on répond volontiers par la négative à la question de la prédicabilité de l'existence chez Leibniz : l'existence n'est pas un prédicat, dit-on, car elle n'est pas une perfection, mais plutôt une relation, comme nous venons de le voir ${ }^{28}$. C'est juste, mais l'affaire est plus délicate. Quelques difficultés subsistent dans les textes eux-mêmes : il semble parfois faire peu de doute que l'auteur utilise l'existence comme un prédicat, ce qu'il déclare même explicitement à certains endroits. Ainsi peut-on lire, dans les Nouveaux Essais, que « lorsqu'on dit, qu'une chose existe, ou qu'elle a l'existence réelle, cette existence même est le prédicat, c'est-à-dire, elle a une notion liée avec l'idée dont il s'agit, et il y a connexion entre ces deux notions $»^{29}$. On objectera que l'auteur ne fait ici que répondre à Locke qui, ayant défini dans son Essay la connaissance comme la perception de la convenance ou de la convenance entre deux idées (IV, I, §1), distingue entre quatre espèces de convenance ou disconvenance, dont la troisième est «Coexistence, ou connexion nécessaire » (IV, I, §3). Quoiqu'il en soit, il ne fait aucun doute que " existant» est traité comme un prédicat de premier ordre dans les fragments logiques, notamment en Recherches générales, §71, comme le note J.-B. Rauzy $^{30}$. Il serait donc faux de dire que, chez Leibniz, l'existence n'est en aucun cas considéré comme un prédicat.

Il faudrait en somme distinguer deux approches : l'existence est un prédicat au sens logique du terme, mais ne l'est pas au sens métaphysique ${ }^{31}$. Et c'est exactement ce que Kant fera, en distinguant le prédicat logique du prédicat réel, lorsqu'il regrettera que "l'illusion qui naît de la confusion d'un prédicat logique avec un prédicat réel (c'est-à-dire avec la détermination d'une chose) repousse presque tout éclaircissement. Tout peut servir à volonté de prédicat logique jusqu'au sujet qui peut se servir à lui-même de prédicat, car la logique fait abstraction de tout contenu. Mais la détermination est un prédicat qui s'ajoute au concept du sujet et l'augmente. Elle ne doit donc pas y être déjà contenue ${ }^{32}$. Voilà qui confirme une lecture kantienne de Leibniz : la position leibnizienne préfigure de manière flagrante celle de Kant. Ainsi peut-on conclure définitivement sur la question de savoir si l'existence est une perfection ou, plus largement, un prédicat: parce que l'existence n'est pas une perfection, mais une relation comparative de perfections entre elles, elle n'est pas un prédicat réel, mais reste un prédicat logique.

\section{III- Les définitions de l'existant}

On trouve dans les textes au moins trois définitions de l'existant : l'existant est ce qui est distinctement senti, ce qui plaît à un esprit et ne déplaît pas à l'esprit le plus puissant et, enfin, ce qui est le plus harmonique. La première exige quelques développements examinant la relation de l'existence à l'espace et au temps, d'une part, et à la perception d'autre part.

\footnotetext{
${ }^{28}$ Voir par exemple M. de GAUDEMAR, op. cit., p. 43 : « l'existence n'est pas un prédicat, une perfection, mais un degré supplémentaire de perfection. Il y a progrès dans le passage à l'existence $»$.

${ }^{29}$ Nouveaux Essais, IV, I ; GP V 339, 34, spn.

${ }^{30}$ Rauzy, p. 315, n. 72. Voir aussi Opuscules et fragments inédits de Leibniz, extraits des manuscrits de la Bibliothèque royale de Hanovre, Louis Couturat (ed.), Paris: Alcan, 1903, p. 271 et 375.

${ }^{31}$ C'est également la conclusion à laquelle arrive J. SKOSNIK, « Leibniz and Russell on Existence and Quantification Theory », Canadian Journal of Philosophy, 10:4, 1980, p. 719.

${ }^{32}$ I. KANT, Critique de la raison pure, AK III 400-401, A597-598/B625-626, in Euvres philosophiques, op. cit., p. 1213-1214.
} 


\section{A/ L'existant est ce qui est distinctement senti}

Pourquoi commencer par là ? Car bien qu'elle ne soit pas la seule définition possible, il s'agit de la seule définition claire : il n'y a pas d'autre « notion claire de l'existence que celle d'être sentie $»^{33}$.

L'une des définitions de la possibilité consiste à identifier possible et « pensable de manière distincte $»:$ «J'appelle possible tout ce qui est parfaitement concevable $\rangle^{34}$; «Possibile est quicquid clare distincteque cogitabile est*. Impossible contra ${ }^{35}$; « que ces Mondes soient possibles ou, ce qui est la même chose, intelligibles distinctement ${ }^{36}$; «distincte cogitabile est, sive possibilis ${ }^{37}$. C'est tout à fait parallèlement que Leibniz définit l'existence en identifiant existant et « perceptible de manière distincte $»$ :

« L'ÊTRE est le pensable de manière distincte.

L'EXISTANT est le perceptible de manière distincte. ${ }^{38}$

Ce par quoi l'existence se distingue de la possibilité est donc, au moins en partie, ce par quoi la perception se distingue de la pensée : l'espace-temps. Avant d'examiner la définition proprement dite de l'existence en terme de perception distincte, il convient donc de considérer sa relation, complexe, à l'espace et au temps comme à deux conditions.

\section{1) Existence et espace-temps}

Exister, c'est occuper un espace: "Tout cela existe, en effet, qui occupe un espace quelconque $»^{39}$. Autrement dit, toute existence est « existence dans ». Par ailleurs, on sait que l'occupation de l'espace est ce qui définit le corps : « la définition du corps est d'exister dans l'espace ». Si non seulement tout ce qui est corps existe, mais aussi tout ce qui existe est corps, alors exister, c'est être un corps. Mais Leibniz précise : «Au terme d'existence dans cet espace se rapporte le mouvement, car dès qu'un corps se met à exister dans un autre espace que précédemment, par cela même il se meut ${ }^{40}$, c'est pourquoi " j'ai trouvé qu'un corps au repos n'existe pas et ne diffère pas d'un espace vide $»^{41}$. Autrement dit, exister, c'est être un corps en mouvement.

${ }^{33}$ Texte supposé être de 1666, cité par Foucher de Careil, op. cit., t. I, p. 11.

${ }^{34}$ Lettre à Bourguet de décembre 1714, GP III 573-574, spn.

${ }^{35}$ Vorarbeiten zur Characteristica Universalis. Definitionentafel, 1671-1672, A VI-2 494.

${ }^{36}$ GP V 246, spn.

${ }^{37}$ L. CoUTURAT, op. cit., p. 77, spn. Voir également Lettre à Louis Bourguet, décembre 1714, GP III 573 ; Meditationes de Cognitione, Veritate et Ideis, GP IV 424 ; Discours de métaphysique, XXIV sq., GP IV 449 sq. ; Essais de Théodicée, 26, GP VI 432.

${ }^{38}$ Définitions (1679-1686), in Rauzy, p. 110. «Ens est distincte cogitabile. Existens distincte perceptibile » (Definitiones : ens, possibile, existens, Sommer 1687 bis Ende 1696 (?), A VI-4-A 869).

${ }^{39}$ Lettre à Thomasius du 30 avril 1669, GP I 27, in Leibniz - Thomasius, Correspondance 16631672, R. Bodéüs (ed.), Paris : Vrin, 1993 (ci-après : Bodéüs), VI, p. 101.

${ }^{40}$ Profession de foi de la nature contre les Athées (1668), I, in Leibniz, Discours de métaphysique et autres textes 1663-1689, C. Frémont (ed.), Paris : Flammarion, 2001, p. 29 pour les trois citations.

${ }^{41}$ Lettre à Arnauld de début novembre 1671, in Leibniz, Cuvres choisies, L. Prenant (ed.), Paris : Garnier, 1940, p. 31. Leibniz s'explique : « le mouvement n'est pas à proprement parler donné dans les corps comme une réalité qui serait en eux, mais je l'ai démontré, tout ce qui se meut est créé de façon continue, et les corps sont quelque chose à tout instant assignable entre ces instants pendant le mouvement » (Lettre à Thomasius du 20-30 avril 1669, ibid., p. 21). 
Si donc le statut de l'existence est, comme le note Herrera, " similaire » à celui de l'espace ${ }^{42}$, c'est simplement parce que l'existence se définit en terme d'espace. Reste un paradoxe, et non le moindre : cet espace qui définit l'existence n'est lui-même que possible.

Exister, c'est occuper le temps: " l'existence telle que nous la concevons enveloppe un temps déterminé; nous disons que quelque chose existe seulement lorsque nous pouvons à un certain moment du temps dire "cette chose existe maintenant" $»^{43}$. Que l'existence implique le temps, c'est d'autant plus évident que l'existence se définit comme le fait, pour un corps, d'être en mouvement, car le mouvement implique lui-même le déplacement non seulement dans l'espace mais également dans le temps. La véritable loi de l'existence, en somme, est d'une certaine manière la temporalité en tant qu'elle est succession.

On souligne volontiers le caractère flou de la relation, chez Leibniz, de l'existence et de la temporalité ${ }^{44}$. Rares, effectivement, sont les passages qui en traitent clairement et distinctement. Ce que l'on peut néanmoins assurer, c'est que l'existence implique la temporalité sans pour autant y être identifiée : «Tempus nihil aliud esse quam ipsam rerum existentiam, seu actualitatem, adeoque lapsu temporis etiam existentiam interire et novari. Sed haec assentio rursus probatione indiget $\gg{ }^{45}$. Si Leibniz refuse à cette implication de devenir une équivalence, c'est que l'existence n'implique pas seulement le temps : comme nous l'avons vu, elle implique également l'espace.

Ainsi l'espace et le temps sont-ils les deux conditions de l'existence. S'impliquant mutuellement, comme en témoigne le mouvement, elles sont cumulatives et non concurrentes. Leibniz répartit effectivement les rôles de la façon suivante. Le temps est l'ordre de l'existence, l'espace celui de la coexistence: "Tempus est ordo existendi eorum quae non sunt simul. Spatium est ordo coëxistendi seu ordo existendi inter ea quae sunt simul »; "Spatium nihil aliud est praecise quam ordo coëxistendi, ut Tempus est ordo existendi, sed non simul ${ }^{46}$. Ou, pour le dire autrement, le temps est condition de l'existence du possible, l'espace est condition de l'existence du compossible.

\footnotetext{
${ }^{42}$ A. HERRERA-IBAÑEZ, op. cit., p. 430-431 : «En la ontología de Leibniz, el status de la existencia es similar al del espacio. Ya vimos que tanto la existencia como la situación son para Leibniz cualidades externas $»$.

${ }^{43}$ Mon principe (décembre 1676), in Rauzy, p. 29. Trois siècles plus tard, Russell posera le problème dans les mêmes termes : "Il convient de réserver le terme d'existence aux choses qui sont dans le temps, c'est-à-dire, qui sont telles que nous pouvons indiquer un moment du temps où elles existent (sans exclure la possibilité qu'elles existent tout le temps). C'est ainsi que les pensées, les sentiments, les esprits et les objets physiques existent. En ce sens, les universaux n'existent pas ; nous dirons qu'ils subsistent ou possèdent l'être, "l'être» étant opposé à 《l'existence» en tant qu'intemporel. Le monde des universaux peut donc aussi être appelé le monde de l'être; monde immuable, rigide, exact, joie du mathématicien, du logicien, du constructeur de systèmes métaphysiques et de tous ceux qui préfèrent la perfection à la vie. Le monde de l'existence, lui, est changeant, vague, sans délimitations bien nettes, sans ordre ni arrangement manifeste ; mais il contient les pensées et les sentiments, les données des sens, les objets physiques, bref tout ce qui peut faire du bien ou du mal, tout ce qui compte en termes de valeur de l'existence et du monde » (Russell, Problèmes de philosophie, IX, F. Rivenc (ed.), Paris : Payot, 1989, p. 123-124). Leibniz, quant à lui, n'oppose pas exister et subsister (voir B. MATES, op. cit., p. 177-178).

${ }^{44}$ M. de GAUDEMAR, op. cit., p. 42 : «Elle a un rapport avec la temporalité, et avec la mise en acte ; mais ce n'est pas encore exactement cela ».

${ }^{45}$ Animadversiones ad Weigelium, in Leibniz, Lettres et Opuscules inédits de Leibniz, Foucher de Careil (ed.), Paris : Ladrange, 1854, p. 158.

${ }^{46}$ Respectivement GM VII 18 et GP III 612.
} 
Le temps, chronologiquement, est donc une condition plus «primaire » que l'espace, qui n'intervient qu'avec la compossibilité, et qui présuppose donc, avant d'examiner la coexistence des possibles candidats, que le critère temporel soit déjà satisfait. Mais, à l'inverse, le temps, logiquement, est une condition plus " secondaire » en ce qu'elle n'examine que la partie (l'existence particulière de chacun des possibles) du tout (l'ensemble des coexistences) : « Pour moi, j'ai marqué plus d'une fois, que je tenais l'Espace pour quelque chose de purement relatif, comme le Temps; pour un ordre des Coexistences, comme le temps est un ordre de successions. Car l'espace marque en termes de possibilité un ordre des choses qui existent en même temps en tant qu'elles existent ensemble, sans entrer dans leurs manières d'exister particulières ${ }^{47}$.

L'espace et le temps, comme conditions de l'existence, sont alors a priori. Dire que le temps est l'ordre de l'existence et l'espace celui de la coexistence est dire qu'ils constituent ensemble et de manière complémentaire le cadre a priori de l'existence, ou les « formes a priori de toute mondanité possible » ${ }^{48}$, pour utiliser une formule qui rappelle combien, sur ce point, Leibniz est précurseur de Kant. Ce caractère formel est mis en évidence par l'hypothèse que, sans les choses qu'ils encadrent, l'espace et le temps ne seraient jamais que de pures idéalités, c'est-à-dire de pures possibilités : «Ainsi je n'admets point ce qu'on avance ici, que si Dieu seul existait, il y aurait temps et espace comme à présent. Au lieu qu'alors à mon avis, ils ne seraient que dans les idées, comme des simples possibilités »; "le temps sans les choses n'est autre chose qu'une simple possibilité idéale ${ }^{49}$.

Et ce n'est pas dire, pour autant, que l'espace et le temps sont davantage du côté des possibles que de celui des existants; car en tant que condition du passage des uns aux autres, ils s'y rapportent équitablement : « le temps et l'espace vont aussi bien aux possibles, qu'aux existants. (...) C'est comme je viens de dire que le temps et l'espace marquent des possibilités au-delà de la supposition des existences. Le temps et l'espace sont de la nature des vérités éternelles qui regardent également le possible et l'existant $\gg{ }^{50}$. Ils composent, en somme, la continuité de l'un à l'autre : l'espace et le temps sont les conditions englobantes de l'existence, c'est-à-dire également de la possibilité en tant qu'elle est dialectiquement contenue dans l'existence. Ensemble, l'espace et le temps composent ainsi « l'ordre des possibilités de tout un Univers » :

«Je reconnais que le temps, l'étendue, le mouvement et le continu en général de la manière qu'on les prend en Mathématiques, ne sont que des choses idéales, c'est-à-dire, qui expriment les possibilités, tout comme font les nombres. Hobbes même a défini l'espace par phantasma existentis. Mais pour parler plus juste, l'Etendue est l'ordre des coexistences possibles, comme le Temps est l'ordre des possibilités inconsistantes, mais qui ont pourtant de la connexion. Ainsi l'un regarde les choses simultanées ou qui existent ensemble, l'autre celles qui sont incompatibles et qu'on conçoit pourtant toutes comme existantes, et c'est ce qui fait qu'elles sont successives. Mais l'Espace et le Temps pris ensemble font l'ordre des possibilités de tout un Univers, de sorte que ces ordres (c'est-à-dire l'Espace et le Temps) cadrent non seulement à ce qui est actuellement, mais encore à ce qui pourrait être mis à la place, comme les nombres sont

\footnotetext{
${ }^{47}$ GP VII 363.

${ }^{48}$ Selon l'expression de Y. Belaval, Etudes leibniziennes : de Leibniz à Hegel, Paris : Gallimard, 1973, p. 207, à propos de l'espace.

${ }^{49}$ Leibniz à Clarke, cinquième écrit, mi-août 1716, respectivement sur 41, 106, in Leibniz, Correspondance Leibniz-Clarke, présentée d'après les manuscrits originaux des bibliothèques de Hanovre et de Londres, A. Robinet (ed.), Paris : PUF, p. 172, orthographe corrigée par nous (ocpn) ; et sur 15, 55, ibid., p. 151, ocpn.

${ }^{50}$ Nouveaux Essais, II, XIV, 24 ; GP V 140.
} 
indifférents à tout ce qui peut être res numerata. Et cet enveloppement du possible avec l'Existant fait une continuité uniforme et indifférente à toute division. ${ }^{51}$

\section{2) Existence et perception}

De l'espace-temps à la perception, le pas est maintenant aisé. Existe ce qui occupe l'espace et le temps. Or, ce qui occupe l'espace et le temps est précisément ce qui s'offre à notre perception. Donc l'existence peut se définir en terme de perception : existe ce qui peut être perçu. Leibniz ajoutera, dans une définition parallèle à celle de la possibilité (est possible ce qui peut être distinctement conçu), existe ce qui peut être distinctement perçu, ou senti : « de même que l'on explique un être par un concept distinct, de même on explique un existant par une perception distincte $»^{52}$.

Dans le but de montrer qu'existe ce qui peut être senti, Leibniz attaque sur deux fronts, bien qu'il semble en avoir abandonné un. Il est effectivement une note dans laquelle il envisage non pas de démontrer directement que ce qui est senti existe, car c'est proprement indémontrable, mais plutôt que ce qui existe est senti : " Quicquid sentitur existit. Indemonstrabilis. Quicquid existit sentitur. Demonstranda $»^{53}$. Que « ce qui est senti existe» est indémontrable, Leibniz l'expose dans son De modo distinguendi phaenomena realia ab imaginariis. Je dois accepter sans preuve l'existence, d'abord, de l'ego comme res cogitans - et par cela seul, disait Descartes, que l'ego est res cogitans car cogito, ego sum ${ }^{54}$ - et, ensuite, des objets extérieurs qui m'apparaissent par la sensation : «En premier lieu, je juge par la simple perception ou expérience que les choses dont je suis conscient à l'intérieur de moi-même existent sans preuve, c'est-à-dire d'abord moi pensant des choses variées, ensuite lesdits phénomènes variés ou apparitions, qui existent en mon esprit $\gg{ }^{55}$.

Dans les faits, cependant, l'auteur développe bien les deux propositions, sans pour autant qu'il s'agisse jamais de véritables démonstrations. Pour montrer que ce qui existe est senti, c'est-à-dire que l'existence révèle le sensible, il fait de l'existence une qualité sensible et externe: "In cogitando reducuntur omnia ad qualitates sensibiles, tum internas, ut calor frigus lux, tum externas, ut $<$ essentia, existentia, $>$ cogitatio, sensio, nihil, unitas, multitudo, identitas, $<$ diversitas $>$, extensio, duratio, situs ; voluptas, possibilitas, actus : hoc. $\rangle^{56}$.

Et pour montrer que ce qui est senti existe, c'est-à-dire que le sensible révèle l'existence, il procède de deux manières. D’une part, en affirmant d'emblée que la

\footnotetext{
${ }^{51}$ Réponses aux réflexions de Bayle, 1702 ; GP IV 568.

${ }^{52}$ De modo distinguendi phaenomena realia ab imaginariis, in Leibniz, Discours de métaphysique et autres textes 1663-1689, C. Frémont (ed.), Paris : Flammarion, 2001, p. 193.

${ }^{53}$ De conatu et motu, sensu et cogitatione, 1671 (?), A VI-2 282.

54 «Il est très vrai, que nous connaissons notre existence par une intuition immédiate (...)» (Sur l'Essay de M. Locke, GP V 18, 21) ; «Descartes a très bien signalé que la proposition : je pense, donc je suis, est une des vérités premières. Mais il eût été convenable de ne pas négliger les autres vérités du même ordre. (...) Aussi est-on en droit de rapporter toutes les vérités de fait premières à ces deux-ci : Je pense, et des choses diverses sont pensées par moi. D'où il suit non pas seulement que je suis, mais encore que je suis affecté de différentes manières» (Animadversiones in partem generalem principiorum cartesianorum, I, art. 7, in Leibniz, Opuscules philosophiques choisis, P. Schrecker (ed.), Paris : Vrin, 2001, p. 39). Notons au passage que le « ego sum, ego existo » cartésien (Meditationes de prima philosophia, 2, AT VII 25, 12 et AT VII 27,9) égalise être et exister - ce que Leibniz se garde bien de faire. Sur la logique du cogito cartésien, voir notre article «Cogito, ergo sum : induction et déduction », Archives de philosophie, 67:1, p. 51-63.

${ }_{55}^{5}$ De modo distinguendi phaenomena realia ab imaginariis, op. cit., p. 193.

${ }^{56}$ Opuscules et fragments inédits de Leibniz, extraits des manuscrits de la Bibliothèque royale de Hanovre, Louis Couturat (ed.), Paris : Alcan, 1903, p. 281.
} 
sensibilité (ou la perception) n'est autre que la preuve de l'existence: une note de révision des années 1697-1700 précise «Nempe qualitatum simul sumtarum compositum seu conceptibilitas constituit rei essentiam, perceptibilitas (...) existentiam ejus probat $\gg{ }^{57}$. D'autre part, par l'absurde en en tirant la conséquence que tout ce qui n'est pas senti n'existe pas: "Car tout ce qui n'est pas senti n'est pas $»^{58}$. Mais, au même moment, c'est-à-dire aux alentours de 1670 , il se montre également moins catégorique et défend seulement que «l'existence d'une chose qui n'est pas sentie ne doit pas être présumée $(..) »^{59}$. On passe ainsi de l'absence d'existence à l'absence de présomption d'existence : non plus « tout ce qui n'est pas senti n'existe pas », mais «tout ce qui n'est pas senti ne doit pas être présumé existant ».

Immanquablement, la position leibnizienne fait songer à la fameuse formule de Berkeley qui, en 1710 et dans le Treatise concerning the Principle of Human Knowledge, I, §3, annonce qu' « exister, c'est être perçu » (esse est percipi).

Voici un texte, supposé être de 1666, dans lequel Leibniz, près d'un demi-siècle avant Berkeley, définit déjà l'existence par un percipi esse :

\begin{abstract}
« Je vis que celui qui aspire à trouver les principes des choses, devait commencer par la considération de l'existence : je me fatiguai des jours entiers à méditer sur cette notion de l'existence : «ordiendum ab ipsius existentiae consideratione credidi ad principia rerum aspiranti: integros dies fatigari inquirendo in notionem existentiae. " Enfin je trouvai que nous autres hommes nous ne pouvions affirmer que ce que nous sentons, (nous sentons aussi des choses dont nous ressentons les effets et les causes, comme quand on jette une pierre d'une élévation, et que cependant nous n'en voyons pas l'auteur). Les choses que nous avons ressenties auparavant, nous les concilions, ou du moins nous croyons pouvoir le faire. De là vient que nous ne croyons pas aux songes quand nous veillons. Mais je voyais cependant qu'il existait ou devait exister nécessairement quelque chose d'autre dans la nature. Car si j'étais seul au monde et que par supposition je vinsse à être enlevé de ce monde, les choses ne périraient pas pour cela avec moi. Mais j'avais beau imaginer d'autres principes, je croyais que tout cela se rapportait à l'essence et non à l'existence, et que je ne pourrais trouver aucune autre notion claire de l'existence que elle d'être sentie. ${ }^{60}$
\end{abstract}

En 1672, l'auteur semble même revendiquer la découverte d'une telle définition : «Ego cum profundius inquirerem, in naturam non Extensionis tantum, sed et generatim existentiae illud tandem reperisse mihi videor, nihil aliud esse Existere quam Sentiri ${ }^{61}$. Dans les écrits postérieurs, il reformulera sa thèse de différentes manières: "Esse nihil aliud esse quam percipi posse » ${ }^{62}$; "La seule idée de l'existence que nous possédions est que nous concevons que les choses sont senties. (...) S'il n'y avait pas d'être sentant, rien n'existerait ${ }^{63}$.

\footnotetext{
${ }^{57}$ Cette note révise un passage de 1667 qui disait «Qualitates simul sumtae (seu imaginabilitas) constituunt Essentiam, sensibilitas Existentiam " (Nova methodus discendae docendaeque jurisprudentiae, 1667, §33, A VI-1 285 ; Z. 10-12 D, spn, pour la note).

${ }^{58}$ Fragment, 1669-1670 (?), in Leibniz, Euvres choisies, L. Prenant (ed.), Paris : Aubier, 1972, p. 88. Il faut ici prendre le second « est» pour « existe », bien entendu.

${ }^{59}$ Préface aux quatre livres de Marius Nizolius sur les vrais principes et sur la vraie méthode de la philosophie, contre les pseudophilosophes dite Sur le style philosophique de Nizolius (1670), §32, in Leibniz, Euvres choisies, L. Prenant (ed.), Paris : Aubier, 1972, p. 91.

${ }^{60}$ Foucher de Careil, op. cit., t. I, p. 10-11.

${ }^{61}$ Propositiones Quaedam Physicae, 1672 (?), Propositio 30 ; A VI-3 56, spn.

${ }^{62}$ déc. 1675, in Jagodinski, Leibnitiana. Elementa philosophiae arcanae, de summa rerum, Kazan, 1913 (ci-après : Jagodinski), p. 14.

${ }^{63}$ De existentia (1676), in Rauzy, p. 30.
} 
Une difficulté, cependant, surgit. Moins de trente années plus tôt, Descartes, ayant montré à quel point le critère de la sensibilité était trompeur, a dû le suspendre dans l'épochè de sa première Méditation. Dès lors, faire de la sensibilité le critère de l'existence, n'est-ce pas, en somme, et d'un point de vue cartésien, prendre le risque de confondre apparence et réalité, rêve et veille ? Leibniz n'ignore pas ce danger, et en fait même son objet dans le De modo distinguendi phaenomena realia ab imaginariis. Mieux : il l'accentue, en soulignant lui-même que ne sont jamais perçues que les apparences : «nihil denique quam apparentiae percipiuntur " ${ }^{64}$. Il y a là de quoi troubler le lecteur! Il suffit effectivement d'un syllogisme pour conclure que si, d'une part, n'existe que ce qui est perçu et, d'autre part, rien n'est perçu que l'apparence, alors n'existe que l'apparence - proposition gênante et, disons-le tout net, indéfendable et indéfendue par l'auteur. Pour se tirer de là, il convient de comprendre adéquatement cette proposition que « rien n'est jamais perçu que des apparences ».

R. M. Adams en propose une interprétation qui, si nous la prolongeons, s'avère extrêmement intéressante : "This must be understood, I think, as a claim that the object of perception, as such, is merely an appearance, whose existence is not distinct from its being perceived ${ }^{65}$. Dire de l'objet perçu qu'il n'est qu'une apparence n'est autre que rappeler que l'objet n'est pas distinct de sa perception. Si cette lecture est correcte, Leibniz écrit de l'objet en général ce que l'interprétation de Copenhague écrira, trois siècles et demi plus tard, de la particule en mécanique quantique, sous le nom fameux de "problème de la mesure ». Heisenberg montrera effectivement que l'« On ne peut plus du tout parler du comportement de la particule sans tenir compte du processus d'observation. En conséquence, les lois naturelles (...) ne concernent plus les particules élémentaires proprement dites, mais la connaissance que nous en avons $\gg{ }^{66}$. De la même manière nous semble-t-il, parce que chez Leibniz l'existence de l'objet dépend du fait de pouvoir être perçu, on ne peut déjà plus du tout parler de l'objet sans tenir compte du processus de perception : la réalité ne décrit déjà plus un état de chose extérieur, mais notre relation au monde. En cela pouvons-nous comprendre l'affirmation leibnizienne selon laquelle nous ne percevons jamais que des apparences : l'objet perçu est apparent en ce qu'il n'est pas objectif, ou réel au sens de Realität, car il n'est pas indépendant du fait d'être perçu - puisque exister, pour lui, est être perçu.

Le piège, quoiqu'il en soit, reste tendu: faire de la sensibilité le critère de l'existence, n'est-ce pas prendre le risque de confondre apparence et réalité, rêve et veille ? Pour l'éviter, Leibniz aura recours à la même arme que Descartes : la clarté et distinction, non plus de l'idée, mais de la perception en question.

Leibniz précise que sa proposition n'est pas «ce qui est senti existe » mais plutôt " ce qui est clairement et distinctement senti existe »: " Imo non quicquid sentitur existit, sed quicquid clare distincteque sentitur $\gg{ }^{67}$. Une note de révision corrige un morceau de la Nova methodus de 1667 trente années plus tard pour préciser : «Essentiam rei nobis esse conceptibilitatem (seu imaginabilitatem) ejus distinctam,

${ }^{64}$ Corpus non est Substantia sed modus tantum Entis sive apparentia cohaerens, 1689-1690 (?), A VI-4-B 1637.

${ }^{65}$ R. M. ADAMS, op. cit., p. 237.

${ }^{66}$ W. HeisenberG, «Das Naturbild der heutigen Physik », in Max Planck Gesellschaft, Jahrbuch, 1953, p. 35-54; traduction française abrégée par A. E. Leroy, La Nature dans la physique contemporaine, Paris : Gallimard, 1962, p. 18. Pour un prolongement de cette lecture antiréaliste, voir notre article "Réalité, Vérité et Connaissance. Une alternative à la traditionnelle 'Justified True Belief', inspirée de la 'Wirklichkeit' du Manuscrit de 1942 de W. Heisenberg », De Philosophia, University of Ottawa, vol. XVI-1, 2000, p. 91-112.

${ }^{67}$ A VI-2 282. 
Existentiam ejus perceptibilitatem (seu sensibilitatem) distinctam ${ }^{68}$. « distinctement» est l'adverbe le plus répandu: «L'EXISTANT est le perceptible de manière distincte ${ }^{69}$; «Existens est quod distincte percipi potest ${ }^{70}$. Mais l'on trouve également «congrument», comme c'est le cas dans le fragment parisien du 15 avril 1676 cité ci-dessous, et le mot a son importance puisqu'il appelle la compossibilité, "Congruum est quod facile compossibile est », et la convenance: " Congruum est quod ita conveniens est, ut nihil addi nec demi opus sit»-les deux étant étroitement liés : «Conveniens est quod facile coexistit seu valde compossibile est $»^{71}$.

Que l'enjeu d'une telle précision soit effectivement d'utiliser, comme Descartes, la distinction comme critère pour reconnaître la réalité de l'apparence ou du rêve, cela se peut voir aisément dans le De modo distinguendi phaenomena realia ab imaginariis et, de manière plus explicite, dans le texte suivant :

\begin{abstract}
« Re recte expensa tantum illud certum est nos sentire, et sentiri congruenter et quandam in sentiendo observari à nobis regulam. Congruenter sentiri est ita sentiri, ut omnium ratio reddi possit, omniaque praedici. Et in hoc consistit existentia, in sensu certas leges servante, nam alioqui omnia essent velut somnia. (...) Porro necesse non est realitate differe quadam intrinseca somnum a vigilia, sed tantùm forma sive ordine sensionum ; unde non est, cur quaeramus an sint quaedam corpora extra nos (...). Ex his patet tantum abesse, ut res materiales sint realiores aliis, ut contra de earum existentia semper possit dubitari aut potius nihil differt materialiter, seu in se, eorum existentia ab existentia somniorum, etsi scilicet pulchritudine differant. $)^{72}$
\end{abstract}

H.-N. Castañeda, qui en livre un commentaire, replace justement cet extrait dans son contexte, celui d'une période grandement influencée par le Théétète de Platon et les Meditationes de Descartes ${ }^{73}$. L'utilisation de l'outil cartésien de la clarté et distinction - certes non plus de l'idée, mais de la perception - est un signe de plus que Leibniz, ici, répond sans doute sur son terrain aux Meditationes de son prédécesseur.

Non plus ce qui est senti, donc, mais ce qui est clairement et distinctement senti. Cette précision est une rectification : elle vient corriger, postérieurement, l'énoncé antérieur et général selon lequel « ce qui est senti existe ».

La perception distincte est le critère de l'existence : elle est nécessaire. Mais elle n'est pas suffisante : Leibniz le rappelle de deux manières.

La perception n'est pas indépendante des autres opérations de l'esprit, elle implique derrière elle, et pour traiter l'information qu'elle donne, la raison, c'est-àdire la réflexion : «Il semble que les sens ne sauraient nous convaincre de l'existence des choses sensibles sans le secours de la raison. Ainsi je croirais que la considération de l'existence vient de la réflexion ${ }^{74}$.

\footnotetext{
${ }^{68}$ Nova methodus discendae docendaeque jurisprudentiae, §33, A VI-1 285, Z. 10-12 D, spn (la note de révision est datée de 1697-1700).

${ }^{69}$ Définitions, in Rauzy, p. 110.

${ }^{70}$ De ente, existente, aliquo, nihilo et similibus, A VI-4-A 570.

71 Vorarbeiten zur Characteristica Universalis. Definitionentafel, 1671-1672, pour les trois citations, respectivement A VI-2 492, 497 et 492.

${ }^{72}$ De veritatibus, de Mente, de Deo, de Universo, 15 avril 1676, A VI-3 511, spn.

${ }^{73}$ Quoiqu'en dise l'auteur : «C'est pourquoi, je ne crains pas de le dire, j'approuve plus de choses

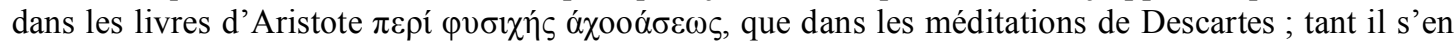
faut que je sois cartésien » (Lettre à Thomasius, du 20-30 avril 1669, in Bodéüs, op. cit., p. 99).

${ }^{74}$ Nouveaux Essais, II, VII ; GP V 117, 1.
} 
La perception est un processus qui s'inscrit dans la continuité des étapes qui mènent de la possibilité à l'existence. Or, il est possible de montrer comment chacune de ces étapes a Dieu comme condition - y compris l'existence elle-même, causée par la volonté divine: "Son entendement est la source des essences, et sa volonté est l'origine des existences » ${ }^{75}$; «Il est la source des possibilités comme des existences, des unes par son essence, des autres par sa volonté $\gg{ }^{76}$. Si donc la perception est le critère de l'existence causée par la volonté divine, c'est qu'elle ne peut elle-même échapper à un rattachement à Dieu. Comment Leibniz procède-t-il pour en témoigner?

En affirmant la thèse qu'exister, c'est être senti par Dieu : «J'en conclus : que l'existence des choses consiste à être sentie par un esprit infaillible dont nous ne sommes que les effluves, c'est-à-dire par Dieu ${ }^{77}$. Si, maintenant, l'on met en parallèle cette définition et celle selon laquelle exister, c'est être distinctement senti (par nous), nous pouvons produire deux conclusions. D'une part, Dieu, lui, n'a pas besoin de l'adverbe « distinctement», car tout ce qu'il sent est par définition distinct. D'autre part, si exister est à la fois être distinctement senti (par nous) et simplement senti par Dieu, et si ce dénominateur commun qu'est l'existence s'efface dans l'équation ainsi obtenue, l'on obtient une équivalence entre sentir distinctement, pour nous, et sentir simplement, pour Dieu : c'est dire, en somme, que le critère de la clarté et de la distinction de notre perception est ce qui nous fait sentir comme Dieu - avec toute la prudence qu'exige une analogie si insolente. Non que nous sentions dès lors avec le même degré de perfection que lui, car la différence sera toujours de nature et non de degré, mais que la clarté et la distinction de notre perception, critère de l'existence des choses, est alors une trace de la perception divine, la marque de l'ouvrier sur son ouvrage. De la même manière que la prétention à l'existence (existentiabilité) est la marque de la nécessité d'exister divine, de la même manière la clarté et la distinction de notre perception qui nous fait reconnaître l'existence des choses, est la marque de la perfection de la perception divine qui n'est autre que la cause de cette existence (exister, c'est être senti par Dieu).

Leibniz, pour mieux s'expliquer, établit deux équivalences. Etre senti par Dieu, dit-il, c'est lui plaire, c'est être harmonieux : «Ego cum profundius inquirerem, in naturam non Extensionis tantum, sed et generatim existentiae illud tandem reperisse mihi videor, nihil aliud esse Existere quam Sentiri, sentiri autem si non a nobis saltem $\mathrm{ab}$ Autore rerum, a quo sentiri, nihil aliud est, quam ei placere, seu Harmonicum esse ${ }^{78}$. Si donc exister est être senti par Dieu, c'est du même coup et également lui plaire et être harmonieux. Développons cela.

\section{B/ L'existant est ce qui plaît à un esprit et ne déplaît pas à l'esprit le plus puissant}

« (...) l'existant est ce qui plaît à un être intelligent et puissant; mais on présuppose alors l'exister lui-même. On pourra cependant donner au moins cette définition : est existant ce qui plairait à un esprit et ne déplairait pas à un autre esprit, plus puissant, si l'on pose que certains esprits existent. On est donc conduit à dire qu'existe ce qui ne déplairait pas à l'esprit le plus puissant, si l'on pose que l'esprit le plus puissant existe. Mais, pour que cette définition puisse être appliquée à l'expérience, on doit plutôt la formuler ainsi : EXISTE ce qui plaît à un esprit (existant) (on ne peut pas ajouter existant si l'on

\footnotetext{
${ }^{75}$ Théodicée, I, §7, GP VI 106.

${ }^{76}$ Nouveaux Essais, II, 15, GP V 141.

${ }_{77}^{77}$ Texte supposé être de 1666, cité par Foucher de Careil, op. cit., t. I, p. 11.

${ }^{78}$ Propositiones Quaedam Physicae, 1672 (?), Propositio 30 ; A VI-3 56.
} 
recherche une définition et non une simple proposition) et qui ne déplaît pas (absolument) à l'esprit le plus puissant. ${ }^{79}$

Leibniz construit ici une définition de l'existence en trois couches.

La première couche repose elle-même sur une hypothèse préalable : "certains esprits existent ». Elle s'énonce alors : « est existant ce qui plairait à un esprit et ne déplairait pas à un autre esprit, plus puissant $»$. Il $\mathrm{y}$ a donc à ce stade deux conditions cumulatives de l'existence: plaire à un esprit quelconque, d'une part, et ne pas déplaire à un autre esprit, plus puissant que le premier, d'autre part. Notons la double négation de la formule "ne pas déplaire », qui logiquement se ramène à "plaire " mais dénote quelque chose de plus : cet esprit plus puissant a la faculté d'annuler les prétentions du candidat à l'existence si (et par ce seul fait) celui-ci ne lui plaît pas. En d'autres termes : le fait de plaire à un esprit quelconque est une condition certes nécessaire mais insuffisante de l'existence, tandis que le fait de déplaire à un esprit plus puissant serait, cette fois, une condition nécessaire et suffisante de l'inexistence. L'existence, en somme, est laissée à la discrétion de l'esprit le plus puissant des deux.

La deuxième couche est la version singulière et superlative de la première. L'hypothèse préalable n'est plus «certains esprits existent» mais «l'esprit le plus puissant existe ». Le superlatif implique effectivement la singularité. L'existence, ellemême, est un maximum, et il n'y a jamais qu'un seul maximum ${ }^{80}$. Cette deuxième couche s'énonce alors: est existant «ce qui ne déplairait pas à l'esprit le plus puissant ». Leibniz n'a fait ici que pousser la seconde condition de l'existence jusqu'à son extrémité, en passant du comparatif ("plus puissant que le premier») au superlatif («le plus puissant»), afin d'embrasser tous les cas et d'énoncer, de la sorte, une proposition absolue et non plus seulement relative. Reste que cette deuxième couche, en l'état, est incomplète puisqu'elle oublie la première condition, qui était que l'existant plaise aussi à un esprit quelconque. En droit, dans l'absolu, l'esprit le plus puissant n'a certes pas besoin d'un esprit quelconque pour faire passer à l'existence mais, et c'est là une objection très anti-réaliste au sens contemporain du terme ${ }^{81}$, en fait, relativement à nous, l'existant autorisé seulement par lui serait à proprement parler inconnaissable donc, en un sens, inexistant, pour nous. Aussi faut-il rétablir la première condition à côté de la seconde, même dans sa version superlative, pour que «cette définition puisse être appliquée à l'expérience ». De cette manière l'auteur appelle une troisième couche.

${ }^{79}$ Recherches générales sur l'analyse des notions et des vérités, 73, in Rauzy, p. 247-249. GP I 73.

${ }^{80}$ J. Moreau, L'univers leibnizien, Paris: Vitte; réed. 1987: Olms, p. 203 : l'existence « correspond au maximum de détermination ou d'essence. Or, ce maximum n'est atteint que dans l'individualité ».

${ }^{81}$ Contrairement à l'interprétation réaliste de J. Bouveresse, pour qui « Il est clair que le possible et l'impossible, chez Leibniz, sont des propriétés objectives des choses ou des états de choses, indépendantes de nos capacités de concevoir et d'imaginer » (J. BOUVERESSE, « La théorie du possible chez Descartes », Revue internationale de philosophie, 37:146, p. 294), il nous semble possible de défendre une interprétation anti-réaliste, que partagerait sans doute Deleuze : « Le monde n'existe pas en soi, le monde, c'est uniquement l'exprimé. Le monde entier est contenu dans chaque notion individuelle, mais il n'existe que dans cette inclusion. Il n'a pas d'existence au dehors. C'est en ce sens que Leibniz sera souvent, et pas à tort, du côté des idéalistes : il n'y a pas de monde en soi, le monde n'existe que dans les substances individuelles qui l'expriment » (cours sur Leibniz du 15 avril 1980); «Il ne faut pas dire que chaque sujet est un miroir sur le monde, parce que ça aurait l'air de dire que le monde existe en soi. Or il n'existe - rappelez vous -, il n'existe que comme plié, c'est à dire il n'existe que enfermé dans chaque âme, il n'existe que enveloppé dans chaque âme ou sujet. (...) En d'autres termes le monde n'existe pas hors des sujets qu'il inclue, le monde n'existe pas hors des sujets qu'il enveloppe » (cours sur Leibniz du 29 avril 1980). 
La troisième et dernière couche, en effet, réunit la première condition de la première couche et la deuxième condition de la deuxième couche. L'hypothèse préalable habituelle, à savoir l'existence actuelle de l'esprit quelconque en question, qui doit bien exister lui-même avant de juger le candidat à l'existence, n'est pas nécessaire dans cette version définitive s'il s'agit d'une définition (mais le serait si l'on faisait une proposition). Cette dernière couche s'énonce : est existant «ce qui plaît à un esprit [...] et qui ne déplaît pas (absolument) à l'esprit le plus puissant ». On retrouve donc, et cette fois définitivement, nos deux conditions cumulatives : le fait de plaire à un esprit quelconque et le fait de ne pas déplaire à l'esprit le plus puissant, c'est-à-dire Dieu. En somme, de ce qui nous plaît, de ce que nous pouvons concevoir, n'existe que ce qui ne déplaît pas à Dieu, qui vient confirmer ou infirmer l'existence, jouant ainsi le rôle de l'agent compossibilateur, qui donne l'existence en donnant son accord.

\section{C/ L'existant est ce qui est le plus harmonique}

Exister, écrit Leibniz, n'est rien d'autre qu'être harmonique : « Existere nihil aliud esse, quam Harmonicum esse ${ }^{82}$. C'est, peut-on ajouter, participer à l'Harmonie universelle, qui commande le passage à l'existence de chaque possible en estimant leur caractère harmonique, c'est-à-dire, littéralement, leur «maximum compossible» ${ }^{83}$ - l'existant étant le compossible parfaitement, «Existens compossibile perfectissimo ${ }^{84}$. Aussi l'existence du maximum possible d'essence n'est-il rien d'autre que l'Harmonie universelle : « Recte expensis rebus pro principio statuo Harmoniam rerum, id est quantum plurimum essentiae potest existat. Sequitur plus rationis esse ad existendum quam ad non existendum. Et omnia extitura si id fieri posset $\gg{ }^{85}$.

Mais chaque possible n'est-il pas déjà harmonique ? La correspondance Leibniz / Wolff identifie effectivement degré d'essence et degré d'harmonie ${ }^{86}$, et l'interprétation de O. Koistinen et A. Repo exige, par exemple, que tous les mondes possibles soient harmoniques: « (...) our interpretation requires that all possible worlds are harmonious : without harmony there is no being in the same world ${ }^{87}$. Dès lors, l'harmonie est-elle condition de l'existence, ou de la simple possibilité ? Si exister est davantage qu'être possible, et qu'être possible est déjà être harmonique, alors exister n'est-il pas davantage qu'être harmonique ? La difficulté n'est qu'apparente, car elle oublie l'un des principes fondamentaux de la discussion : il n'y a jamais entre possibilité et existence qu'une différence de degré, aussi ne peut-on contenir l'harmonie dans l'un sans qu'elle s'écoule dans l'autre. L'existence, pour se distinguer, n'a alors pas besoin d'être davantage qu'harmonique (ce qui n'est pas même possible), il lui suffit d'être davantage harmonique : l'existant, qui est le maximum compossible, est du même coup le maximum harmonique. Exister, c'est donc être le plus harmonique : «le terme qui est élu est le plus harmonique:

\footnotetext{
${ }^{82}$ Jagodinski, op. cit., p. 32.

${ }^{83}$ Comme dit M. FiCHANT, «'Pour la beauté et pour l'harmonie' : le Meilleur de la Dynamique », in A. Heinekamp et A. Robinet (ed.), Leibniz : le meilleur des mondes, Studia Leibnitiana, Sonderheft 21, Stuttgart : Steiner, 1992, p. 235.

${ }^{84}$ Definitiones : ens, possibile, existens, Sommer 1687 bis Ende 1696 (?), A VI-4-A 867.

${ }^{85}$ De arcanis sublimium vel de summa rerum, 11 février 1676, A VI-3 476.

${ }^{86}$ Briefwechsel zwischen Leibniz und Christian Wolf, Gerhardt (ed.), Halle, 1860, p. 172.

${ }^{87}$ O. KoIstinen \& A. REPO, "Compossibility and Being in the Same World in Leibniz's Metaphysics », Studia Leibnitiana, 31:2, 1999, p. 211-212.
} 


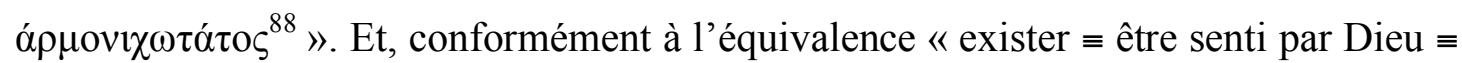
lui plaire $\equiv$ être harmonique au plus haut point », est harmonique au plus haut point ce qui est le plus plaisant pour l'esprit le plus parfait, comme le rappelle ce morceau parisien : « Harmonicum maxime quod gratissimum [perfectissimae] mentium ${ }^{89}$.

Récapitulons. L'existence est bien un degré de possibilité, un ajout, un complément, mais n'ajoute rien de nouveau. Elle n'est pas une perfection, mais une relation comparative de perfections entre elles. Elle n'est pas un prédicat réel, mais peut être considérée comme un prédicat logique. Elle se définit comme ce qui est distinctement senti, ce qui plaît à un esprit tout en ne déplaisant pas à l'esprit le plus puissant, et ce qui est le plus harmonique. Pour une notion primitive a priori indéfinissable, inanalysable et simple, l'existence leibnizienne apparait a posteriori définissable, analysable, et surtout complexe. Sa nature, ses attributs et ses définitions sont pour le moins hétérogènes. Elle peut s'appréhender par différents chemins, qui sont autant de modes exprimant une substance unique. Riche de ces différentes couches, l'existence se replie dans le cercle de l'ontologie leibnizienne qui la fond dans la possibilité. Cette relation complexe de la possibilité à l'existence, par l'existentiabilité, garde de nombreux mystères. Ce qui est certain est que Leibniz n'a pas attendu Kant pour poser, dans toute sa force, le problème de l'existence ${ }^{90}$.

${ }^{88}$ A. RIVAUD, « Textes inédits de Leibniz publiés par M. Ivan Jagodinski », Revue de métaphysique et de morale, 22:1, 1914, p. 94-120, renvoie en note à "Hannover, Königl. Bibliothek, LeibnizHandschriften, Abth. 37 (Physik), Vol. V, f. 222-223 ; 222 recto : In Mente omnes conatus durant, nec

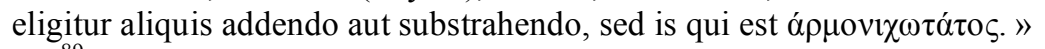

${ }^{89}$ De arcanis sublimium vel de summa rerum, 11 février 1676, A VI-3 476.

${ }^{90}$ Pour un prolongement de cette discussion chez Kant, voir notre article « De la possibilité à l'existence : Kant critique de Leibniz », Dialogue : Revue canadienne de philosophie, à paraître. 